\title{
Density of optical degrees of freedom: intensity, linear and angular momentum
}

\author{
Michael Mazilu \\ SUPA, School of Physics and Astronomy, \\ University of St Andrews, St Andrews, United Kingdom
}

\begin{abstract}
For any optical system, optical eigenmodes describe solutions of Maxwells equations that are orthogonal to each other. In their simplest free space form, these modes correspond, for example, to Bessel, Laguerre-Gaussian or Hermite-Gaussian beams. However, the orthogonality property is not limited to the intensity of the optical field but more generally the optical eigenmode decomposition can be applied to the linear and angular momentum arising from complex coherent beams. These modes can be seen as describing the independent degrees of freedom of the optical system and are characterized by the mode, their density and coupling efficiency. It is interesting to study the effect of different optical systems on the density of the optical degrees of freedom propagating through them. Here, we look at systems containing different elements such as: dielectric, meta-material and random lenses. Using the optical eigenmode decomposition, we determine their density in these different cases and discuss the origin of the variations observed. Further, we study the overall number of optical degrees of freedom accessible including linear and angular momentum of optical beams.
\end{abstract}

Keywords: optical eigenmodes, structured illumination, optical degrees of freedom

\section{INTRODUCTION}

Many applications in optics rely on the capability to create or detect beams having a specific profile. Generally, this capability depends on the number of optical degrees of freedom accessible by the source, transmitted by the optical system and finally detectable by the measuring device. In this paper, we study how the optical system affects different definitions of the optical degrees of freedom (ODOF). The total number of ODOF gives us insight into how many independent channels can be used for information transmission in an optical system. Indeed, each ODOF corresponds to an independent and orthogonal electromagnetic field that can used as an independent communication channel. ${ }^{1}$ Introducing the concept of density of ODOF we can asses the optical imaging resolution of the system. ${ }^{2,3}$ In this case, the higher the density the smaller the point spread function of the optical system and ultimately the higher the resolution of the imaging system. The optical eigenmodes (OEi) offer

Send correspondence to: michael.mazilu@st-andrews.ac.uk

Complex Light and Optical Forces VIII, edited by David L. Andrews, Enrique J. Galvez, Jesper Glückstad, Proc. of SPIE Vol. 8999, 89990U - @ 2014 SPIE · CCC code: 0277-786X/14/\$18 · doi: 10.1117/12.2039772 
a natural description of the electromagnetic fields propagating through a linear optical system. ${ }^{4}$ These modes correspond to solutions of Maxwell's equations that are orthogonal with respect to a specific measure. ${ }^{5}$ Counting the number of OEi in an optical system corresponds to the number of optical degrees of freedom accessible in this system. Mathematically, this corresponds to the rank of the system.

\section{THEORY}

The optical eigenmode decomposition is based on the definition of a property of the electromagnetic field as a quadratic function of the field. In this paper, we are considering the time averaged intensity of the field as defined by $I_{R O I}=\int_{R O I} d \sigma \mathbf{E}^{*} \cdot \mathbf{E}$ where the integration is done over the region of interest (ROI). Other properties such as the angular momentum ${ }^{6,7}$ or optical forces ${ }^{8}$ can also be considered.

In the following, we are describing the step by step procedure to determine the optical eigenmodes and the degrees of freedom associated with them in the case of illumination by a family of orthogonal incident beams. Considering the intensity measure, we can describe this intensity as a function of the superposition coefficients $a_{i}$ of $N$ probe fields $\mathbf{E}_{i}$. Indeed, considering the total field described by

$$
\mathbf{E}=\sum_{i=1}^{N} a_{i} \mathbf{E}_{i}
$$

we can rewrite the intensity as

$$
I_{R O I}=\sum_{i=1}^{N} \sum_{j=1}^{N} a_{i}^{*} M_{i j} a_{j}
$$

where we define the matrix $\mathbf{M}$ as

$$
M_{i j}=\int_{R O I} d \sigma \mathbf{E}_{i}^{*} \cdot \mathbf{E}_{j}
$$

Practically, the probes are chosen to correspond each to a member taken from an orthogonal family of fields having the same incident intensity on the optical system considered. For example, the probe fields $\mathbf{E}_{i}$ can be chosen to correspond to a member of the LaguerreGaussian or Hermite-Gaussian beams family. Provided each incident probe field is normalised in intensity over the whole transversal plane and that the superposition coefficients $a_{i}$ are chosen such that

$$
\sum_{i=1}^{N} a_{i}^{*} a_{i}=1
$$

then each set of coefficients $a_{i}$ describes an incident beam that is normalised in intensity over the whole transversal plane. For regions of interest that are finite, this property does not hold any more and the intensity is given by $I_{R O I}$. 
The optical eigenmodes are defined as the beam corresponding to superpositions coefficients $a_{i}$ that describe normalised eigenvectors of the matrix operator $M_{i j}$. Further, by construction, the matrix operator $M_{i j}$ is Hermitian

$$
M_{i j}=M_{j i}^{*}
$$

and as such its eigenvectors are orthogonal to each other and its eigenvalues are real. Physically, the eigenvalues correspond to the integrated intensity of the optical eigenmode in the region of interest (ROI). As such, the optical eigenmode having the largest eigenvalue describes a superposition of probe beams that corresponds to the largest intensity achievable in the ROI considering the probes used. Provided, the probes describe a complete set of probes this property transforms into a global property that delivers the absolute maximum achievable in a linear optical system.

This approach can be generalised to consider different linear measures of the optical system. For example, it is possible to replace the intensity by the energy density, Poynting vector, Maxwell's stress tensor, angular or linear momentum. All this quantities are, by their nature, quadratic with respect to the electromagnetic fields and as such it is possible to introduce in each case a Hermitian matrix operator that can be used to decompose the fields into orthogonal optical eigenmodes.

Having described how to decompose an electromagnetic field in a set of non redundant orthogonal fields, we can now proceed to defining the optical degrees of the system by considering the rank of the matrix operators involved in the decomposition. Indeed, each Hermitian matrix operator can be seen as delivering a number of non-zero eigenvalues. The optical eigenmodes corresponding to non-zero eigenvalues describe observable fields with respect to the measure considered, however, eigenmodes having negligible small eigenvalues describe the null-space of this operator and as such are not observable as an independent degree of freedom. In the following, we use a soft threshold to define whether an eigenvalue is negligible or not i.e. the null-space is defined by the eigenvalues whose absolute value are smaller than a given fraction of the maximal absolute eigenvalue measured. Physically, this approach accounts for a fixed dynamic range in the measurement procedure. The smallest measure observable is defined in relative terms with respect to the largest possible measure. Alternatively, this definition can be described in absolute terms.

\section{MODELLING}

To illustrate the effect of different optical systems on the number and density of the optical degrees of freedom accessible in the ROI, we can use finite element method programs to simulate the propagation of monochromatic light field through simple optical systems. Here, we use Comsol electromagnetic module to consider the propagation of fields in a 2D domain surrounded by perfectly matched layers (PML). The incident fields are defined by the electric field on the left hand side of the rectangular domain (see figure 1), propagate from left to right and are detected on the right hand side interface before the perfectly matched layer. 

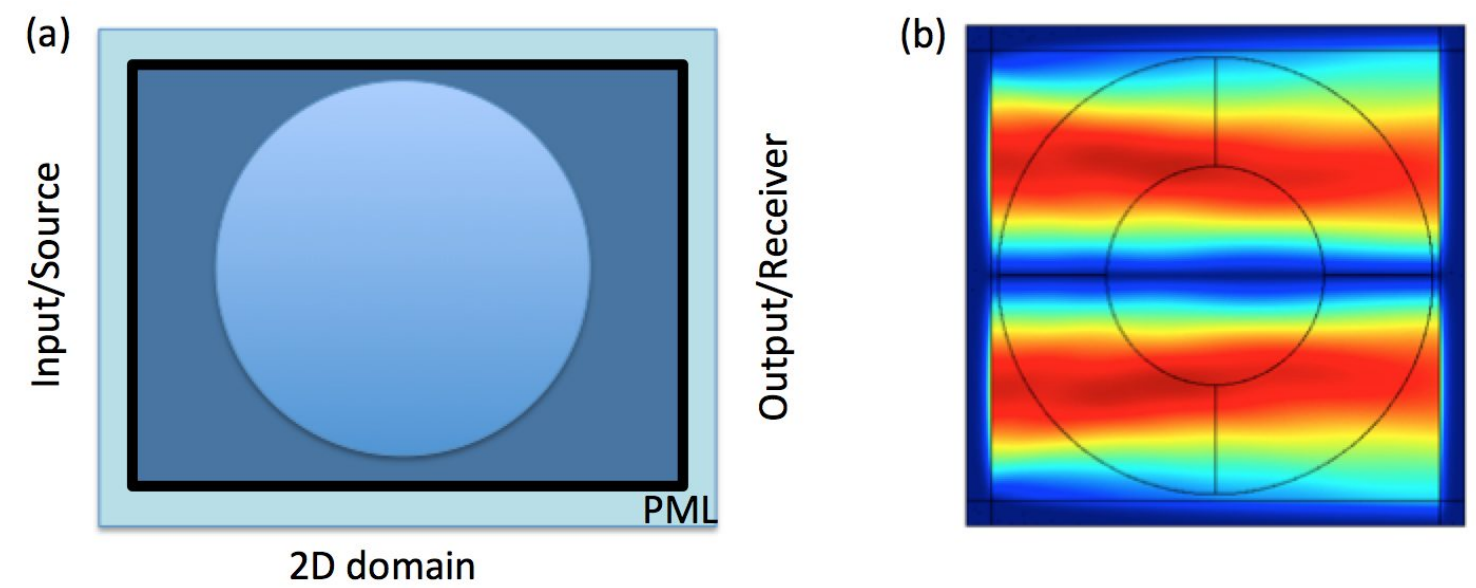

Figure 1. (a) Simulation configuration showing the input and output sides and the optical system having an cylindrical form here. (b) Example probe field in absence of any optical system.

(a)
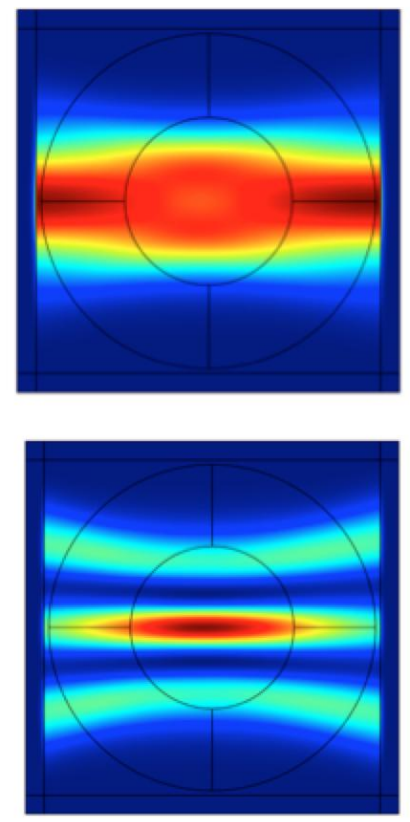
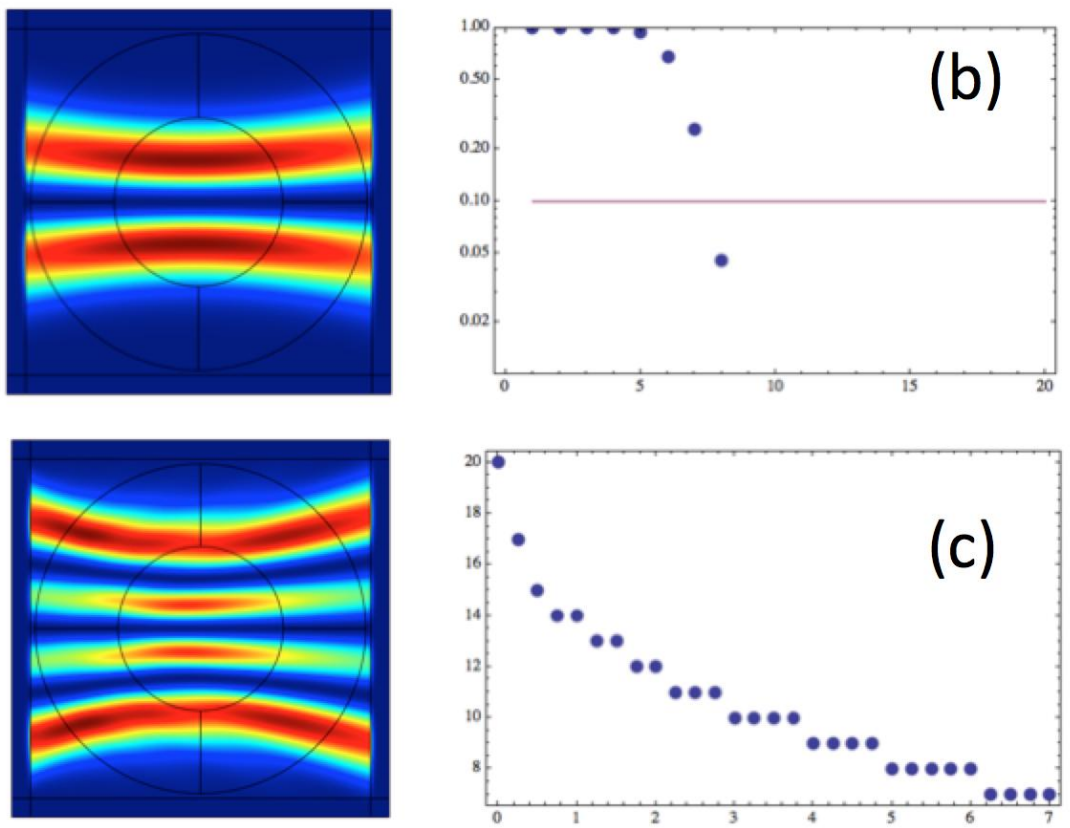

Figure 2. (a) First four optical eigenmodes of free space The receiver plane is on the right hand side and the source plane on the left hand side. (b) Normalised eigenvalue spectrum in the same configuration. The red horizontal line shows the relative null space limit. Eigenvalues above the line are considered as independent degrees of freedom (ODOF) contributing to the rank of the matrix. (c) The number of degrees of freedom as a function of the distance between the source and receiver plane in free space. 


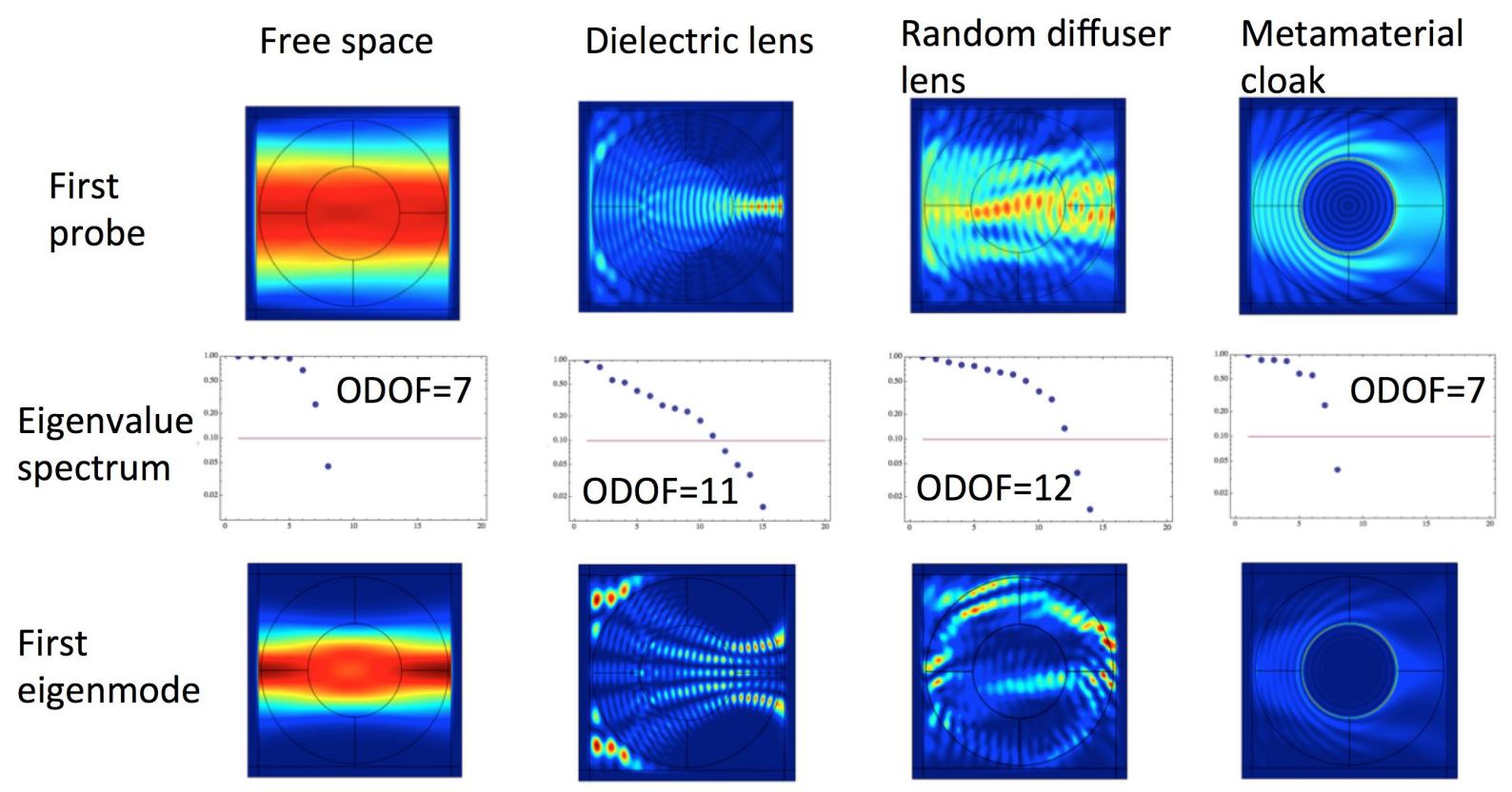

Figure 3. Optical eigenmodes for different optical systems. From left to right: Free space, cylindrical lens, random index lens and metamaterial invisibility "cloak". The top raw show the propagation of the first probe beam through the system, the second raw the spectra of the eigenvalues for each different configuration and the third raw the first optical eigenmode.

\section{RESULTS AND DISCUSSION}

Figure 2 a shows the first optical eigenmodes for free space propagation between the input and output planes. Figure $2 \mathrm{~b}$ shows the distribution of the eigenvalues normalised to the first, largest eigenvalue. This allows the easy counting to the rank of the matrix corresponding to the optical degrees of freedom accessible in the system. In this context, eigenvalues that are larger then $10 \%$ of the largest eigenvalue correspond to optical eigenmodes that are observable. In the case of free space, we can study the effect of the distance between the input/source and the output/received ROI on the number of ODOF. Figure 2c shows that this number decreases continuously as this distance increases. This decrease, in the near field is due to the loss of ODOF as the near field waves decrease evanescently, while in the far field the decrease is due to diffraction.

Figure 3 shows example optical systems, their interaction with the first probe and first intensity eigenmode. For each system, we evaluate the number of ODOF received in the output plane. We remark that, in this circumstance, the largest number of orthogonal degrees of freedom is achieved in the case of the random dielectric lens. As could be expected, the meta material cloak delivers similar results as the free space while the dielectric lens clearly improves the number of degrees of freedom accessible in the output plane as it accts like a relay system. 


\section{REFERENCES}

1. R. Piestun and D. A. B. Miller, "Electromagnetic degrees of freedom of an optical system," JOSA A 17(5), p. 892, 2000.

2. J. Baumgartl, S. Kosmeier, M. Mazilu, E. T. F. Rogers, N. I. Zheludev, and K. Dholakia, "Far field subwavelength focusing using optical eigenmodes," Appl. Phys. Lett. 98(18), p. 181109, 2011.

3. S. Kosmeier, M. Mazilu, J. Baumgartl, and K. Dholakia, "Enhanced two-point resolution using optical eigenmode optimized pupil functions," J Opt 13, p. 105707, Sept. 2011.

4. M. Mazilu, J. Baumgartl, S. Kosmeier, and K. Dholakia, "Optical Eigenmodes; exploiting the quadratic nature of the energy flux and of scattering interactions," Opt. Express 19(2), pp. 933-945, 2011.

5. A. C. De Luca, S. Kosmeier, K. Dholakia, and M. Mazilu, "Optical eigenmode imaging," Phys Rev A 84(2), p. 021803(R), 2011.

6. M. Mazilu, "Spin and angular momentum operators and their conservation," J. Opt. A 11(9), p. 094005, 2009.

7. M. Mazilu, "Optical eigenmodes; spin and angular momentum," J. Opt. 13(6), p. 064009, 2011.

8. M. Ploschner, M. Mazilu, T. Cizmar, and K. Dholakia, "Numerical investigation of passive optical sorting of plasmon nanoparticles," Opt. Express 19(15), pp. 13922-13933, 2011. 